\title{
Individual Movement Variability Magnitudes Are Explained by Cortical Neural Variability
}

\author{
(DShlomi Haar, ${ }^{1,2}$-Opher Donchin, ${ }^{3,4}$ and Ilan Dinstein ${ }^{1,2,4}$ \\ ${ }^{1}$ Department of Brain and Cognitive Sciences, ${ }^{2}$ Zlotowski Center for Neuroscience, ${ }^{3}$ Department of Biomedical Engineering, and ${ }^{4}$ Department of \\ Psychology, Ben-Gurion University of the Negev, Beer-Sheva, 8410501 Israel
}

Humans exhibit considerable motor variability even across trivial reaching movements. This variability can be separated into specific kinematic components such as extent and direction that are thought to be governed by distinct neural processes. Here, we report that individual subjects (males and females) exhibit different magnitudes of kinematic variability, which are consistent (within individual) across movements to different targets and regardless of which arm (right or left) was used to perform the movements. Simultaneous fMRI recordings revealed that the same subjects also exhibited different magnitudes of fMRI variability across movements in a variety of motor system areas. These fMRI variability magnitudes were also consistent across movements to different targets when performed with either arm. Cortical fMRI variability in the posterior-parietal cortex of individual subjects explained their movement-extent variability. This relationship was apparent only in posterior-parietal cortex and not in other motor system areas, thereby suggesting that individuals with more variable movement preparation exhibit larger kinematic variability. We therefore propose that neural and kinematic variability are reliable and interrelated individual characteristics that may predispose individual subjects to exhibit distinct motor capabilities.

Key words: fMRI; motor control; motor system; movement variability; neural variability

\section{Significance Statement}

Neural activity and movement kinematics are remarkably variable. Although intertrial variability is rarely studied, here, we demonstrate that individual human subjects exhibit distinct magnitudes of neural and kinematic variability that are reproducible across movements to different targets and when performing these movements with either arm. Furthermore, when examining the relationship between cortical variability and movement variability, we find that cortical fMRI variability in parietal cortex of individual subjects explained their movement extent variability. This enabled us to explain why some subjects performed more variable movements than others based on their cortical variability magnitudes.

\section{Introduction}

Intertrial variability is a fundamental characteristic of human movements (Harbourne and Stergiou, 2009). Variability of specific kinematic components such as movement extent and movement direction is thought to be governed by independent neural processes (Gordon et al., 1994b; Krakauer et al., 2000; van Beers, 2009) according to the demands of the examined motor task (Todorov, 2004; Latash et al., 2007). Although kinematic variability is det-

Received June 13, 2017; revised July 19, 2017; accepted Aug. 5, 2017.

Author contributions: S.H., O.D., and I.D. designed research; S.H. performed research; S.H. analyzed data; S.H., 0.D., and I.D. wrote the paper.

This work was supported by the Israel Science Foundation (Grant 961/14 to I.D.), the Helmsley Foundation (0.D.), and the $A B C$ Robotics Center. We thank llan Shelef and Moti Salti for help in acquiring the fMRI data and Lior Shmuelof for helpful discussions about the manuscript.

The authors declare no competing financial interests.

Correspondence should be addressed to Shlomi Haar, Ben-Gurion University of the Negev, P.0. Box 653, BeerSheva, 8410501 Israel. E-mail: haar@post.bgu.ac.il.

DOI:10.1523/JNEUROSCI.1650-17.2017

Copyright $\odot 2017$ the authors $\quad 0270-6474 / 17 / 379076-10 \$ 15.00 / 0$ rimental for movement accuracy, it is thought to be critical for motor learning (Wilson et al., 2008; e.g., Braun et al., 2009; Teo et al., 2011; Herzfeld and Shadmehr, 2014; Wu et al., 2014).

Intertrial variability is also a fundamental characteristic of neural activity that is apparent in the variable timing and amplitude of neural responses across trials containing an identical stimulus or task (Stein et al., 2005; Faisal et al., 2008; Churchland and Abbott, 2012; Dinstein et al., 2015; Sauerbrei et al., 2015). As with kinematic variability, intertrial neural variability also seems to be important for motor learning, as demonstrated in studies with songbirds (Kao et al., 2005; Ölveczky et al., 2011; Woolley and Kao, 2015) and primates (Mandelblat-Cerf et al., 2009). Given that neural activity generates behavior, one may expect that intertrial variability in the activity of specific neural populations would generate corresponding intertrial variability in specific kinematic components of movement (e.g., movement extent and/or direction).

Studies that have examined this potential relationship have proposed three alternative theories. The first theory proposed 
A

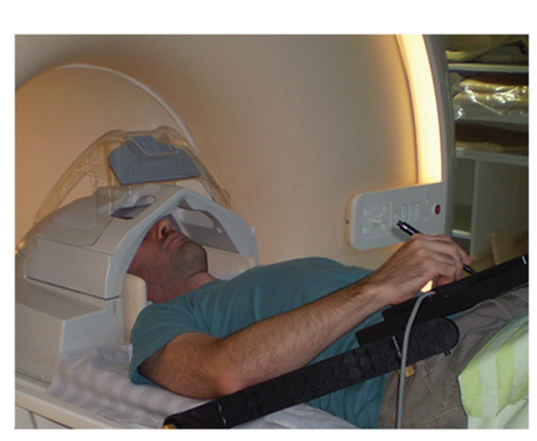

B

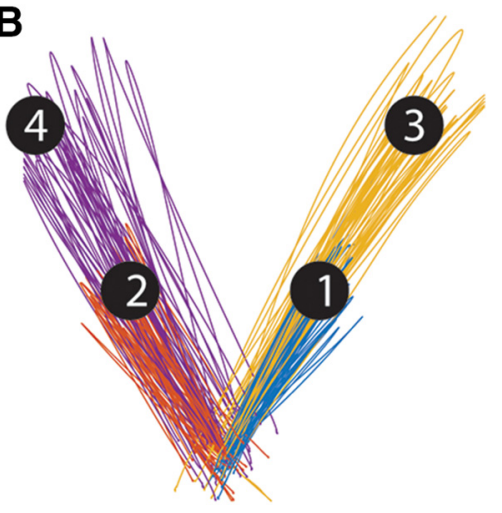

Figure 1. $\quad \boldsymbol{A}$, Experimental setup. $\boldsymbol{B}$, Representative example of movement paths of one subject. Different colors represent slice movements to the four targets.

that kinematic variability during visually guided movements is mostly explained by variability in sensory neural populations. For example, intertrial variability in the initial speed of smooth pursuit eye movements can be explained by variability in the estimation of target speed in MT neurons (Osborne et al., 2005; for review, see Lisberger and Medina, 2015). In contrast, the second theory has proposed that kinematic variability during reaching movements is generated by variable preparatory (motor planning) activity of premotor and primary motor neurons (Churchland et al., 2006). Finally, the third theory has suggested that kinematic variability is caused by neural and neuromuscular variability during actual movement execution (van Beers et al., 2004; van Beers, 2009). Together, these studies suggest that distinct neural variability sources are correlated with kinematic variability under different experimental conditions, which include the sensorymotor requirements of the examined motor task (e.g., smooth pursuit ocular movements vs reaching movements) and the temporal structure of the task (e.g., imposing a delay between movement planning and execution).

In the current study, we examined several outstanding questions regarding kinematic variability, neural variability, and their potential relationship in humans. First, do individual subjects exhibit consistent magnitudes of kinematic variability regardless of the movements that they are performing? Second, do individual subjects exhibit consistent magnitudes of neural variability regardless of the movements that they are performing? And third, if so, are between-subject differences in kinematic variability explained by differences in neural variability in specific sensory and/or motor brain areas? Answering these questions is critical for establishing that individual subjects exhibit characteristic kinematic and neural variability magnitudes that may predispose them to exhibit particular motor learning capabilities while also adding new insights regarding the potential relationship between neural variability and kinematic variability.

To answer the questions above and to relate the findings with the existing behavioral and electrophysiology literature, we quantified intertrial variability of movement direction, peak velocity, and extent across slice (out-and-back) reaching movements. These movements were performed to four peripheral targets with either right or left arm on a touch screen while brain activity was recorded with fMRI. We then quantified fMRI response variability in the primary motor, premotor, parietal, and visual brain areas of each subject and determined whether it was possible to explain between-subject differences in kinematic variability according to neural variability magnitudes in specific brain areas.

Note that, in our study, all movements were performed without visual feedback to preclude the potential influence of neural variability associated with visual input.

\section{Materials and Methods}

Subjects. Thirty-two right-handed volunteers with normal or corrected-to-normal visual acuity (15 women and 17 men, aged 22-36, mean \pm SD $25.6 \pm 2.5$ ) participated in the present study. The Soroka Medical Center Internal Review Board approved the experimental procedures and written informed consent was obtained from each subject. The sample size was selected so that the correlation effect size of 0.4 would have power $>1-\beta=0.75$ (1-tailed test), with $\alpha$ set to 0.05 . According to $\mathrm{G}^{\star}$ Power (Faul et al., 2009), the required minimum sample size is 30 .

Experimental setup and design. Subjects lay in the scanner bore and viewed a back-projected screen through an angled mirror that prevented any visual feedback of their arm and hand. An MRI-compatible digitizing tablet (Hybridmojo) was placed over the subject's waist and used to track his/her arm movements (Fig. 1A). Subjects performed slice (out-and-back) reaching movements from a central target to 4 peripheral targets located 7 and $13 \mathrm{~cm}$ from the central target in each of 2 directions $\pm 45^{\circ}$ from the midline (Fig. $1 B$ ). Subjects did not receive any visual feedback of their arm location during movement. Each trial started with the presentation of a peripheral target for $1 \mathrm{~s}$. Four seconds after the target disappeared, the central target changed from red to green, indicating that the movement should be performed by moving the stylus pen on the tablet. Subjects had 1 s to complete the movement, after which the center target turned red and remained red for the entire intertrial interval (ITI), which lasted $6 \mathrm{~s}$. There was no posttrial visual feedback or knowledge of results. All subjects performed 3 experimental runs with each arm, each of which lasted $9 \mathrm{~min}$ and contained 11 movements to each of the 4 targets in a random order. The experiment started with three runs of the left (nondominant) arm, followed by three runs of the right (dominant) arm. Subjects were trained on the task inside the scanner with both hands, before the scan, until they reported that they were comfortable performing it.

Movement recording and analysis. Kinematic data were recorded at $200 \mathrm{~Hz}$. Trials with a reaction time of $>1 \mathrm{~s}$, trials with a movement angle error $>30^{\circ}$ (at peak velocity or end point), and trials with movement length that was $<50 \%$ or $>200 \%$ of the target distance were discarded from further analysis. Trials containing correction movements (i.e., velocity profiles with more than two peaks) were also removed. On average $\sim 8 \%$ (SD 3\%) of the trials were discarded for each subject. There was no significant difference in the number of discarded trials between the two arms.

We quantified intertrial variability for each of three kinematic components: movement direction, movement extent, and peak movement velocity. Movement extent and peak velocity variabilities were normalized by their respective means in order to compute the coefficient of variation (CV). This was necessary because the variability of these kinematic components scales with their mean (speed-accuracy trade-off; Schmidt et al., 1979). Movement direction variability was quantified by the SD across trials. Each of these measures was computed for each target and each subject separately and then averaged across targets to compute a single extent, peak velocity, and direction variability measure for each subject.

MRI acquisition and preprocessing. Imaging was performed using a Philips Ingenia 3 T MRI scanner located at the Ben-Gurion University Brain Imaging Research Center. The scanner was equipped with a 32 channel head coil, which was used for RF transmit and receive. Blood oxygenation level-dependent (BOLD) contrast was obtained using a $\mathrm{T} 2^{*}$-sensitive echoplanar imaging pulse sequence $(\mathrm{TR}=2000 \mathrm{~ms}$; $\mathrm{TE}=$ $35 \mathrm{~ms} ; \mathrm{FA}=90^{\circ}$; 28 slices; voxel size of $2.6^{\star} 2.6^{\star} 3 \mathrm{~mm}$ and with $0.6 \mathrm{~mm}$ gap). Anatomical volumes were acquired with a T1-weighted sagittal 
sequence $\left(\mathrm{TR}=8.165 \mathrm{~ms} ; \mathrm{TE}=3.74 \mathrm{~ms} ; \mathrm{FA}=8^{\circ}\right.$; voxel size of $\left.1^{\star} 1^{\star} 1 \mathrm{~mm}\right)$.

MRI data were preprocessed with the Freesurfer software package (http://surfer.nmr.mgh.harvard.edu; Fischl, 2012) and FsFast (Freesurfer Functional Analysis Stream). Briefly, this process includes removal of nonbrain tissue and segmentation of subcortical, gray, and white matters based on image intensity. Individual brains were registered to a spherical atlas that used individual cortical folding patterns to match brain geometry across subjects. Each brain was then parcellated into 148 cortical ROIs using the Destrieux anatomical atlas (Destrieux et al., 2010). Functional scans were subjected to motion correction, slicetiming correction, and temporal high-pass filtering with a cutoff frequency of two cycles per scan. Functional scans were registered to the high-resolution anatomical volume. No additional spatial smoothing was performed. Preprocessed data were imported into MATLAB (version R2015a; The MathWorks) and all further analysis was performed using custom software written in MATLAB.

Time course analysis. To ensure that our estimates of intertrial fMRI variability were not generated by head motion, respiration, and blood flow artifacts, we removed the following components from the fMRI time course of each cortical voxel through linear regression: (1) six head motion parameters obtained by rigid body correction of head motion (three translations and three rotations), (2) fMRI time course from the lateral ventricles, and (3) the mean fMRI signal of the entire cortex (i.e., global component). In addition, we normalized the time course of each voxel to a mean of zero and unit variance (i.e., $Z$-score). This ensured that overall time course variance was equal across subjects such that our measure of intertrial fMRI variability captured only task-related trial-by-trial variability differences across subjects rather than variability associated with the entire scanning session.

Identification of ROIs. Visual and motor ROIs in both left and right hemispheres were defined a priori according to a combination of anatomical and functional criteria in the native space of each subject. We first used the automated Freesurfer parcellation pipeline to identify 148 anatomical ROIs in each of the subjects based on the Destrieux anatomical atlas (Destrieux et al., 2010). We then selected the 100 continuous functional voxels that exhibited the strongest activation when contrasting all movement trials versus rest. To confine the ROIs to specific anatomical locations across all subjects we selected the voxels within the following Freesurfer ROIs: early visual cortex (Vis) occipital pole and calcarine sulcus; superior parietal lobule (SPL) anterior portion of the superior parietal lobule superior to the IPS and slightly posterior to the postcentral sulcus; inferior parietal lobule (IPL) dorsal portion of the angular gyrus and the middle segment of the intraparietal sulcus; primary motor cortex (M1) anterior bank of the central sulcus in the hand knob area; dorsal premotor cortex (PMd) junction of superior frontal sulcus and precentral sulcus; ventral premotor cortex (PMv) junction of inferior frontal sulcus and precentral sulcus; and supplementary motor area (SMA) medial wall of the superior frontal gyrus anterior to the central sulcus posterior to the vertical projection of the anterior commissure.

We also defined control ROIs that did not exhibit task-related activations in the dorsolateral prefrontal cortex (dlPFC) middle frontal sulcus and eight ROIs located outside of the brain/head of the subject (one ROI in each corner of the scanned volume). These control ROIs enabled us to demonstrate the specificity of the results to the visuomotor cortices. The choice of dlPFC as a control area was motivated by its proximity to the premotor areas and lack of task-related activity.

Intertrial fMRI variability. Variability across trials was computed for each subject separately relative to their mean hemodynamic response in each ROI. We estimated a hemodynamic response function (HRF) for each subject, ROI, and target by computing the mean response across all trials to a given target. Then, we built a general linear model (GLM) with a row for every time point and a column for every trial. Each column contained a delta function at the time point corresponding to the go cue (movement onset), which was convolved with the HRF described above. This enabled us to estimate a response amplitude ( $\beta$ value) for each trial using multiple regression. Note that, by using individual subject HRFs for this analysis, we were able to entirely discount the mean HRF ampli- tude and shape from our estimates, yielding a pure (isolated) measure of individual intertrial variability relative to the mean.

Intertrial fMRI variability was estimated as the $\mathrm{SD}$ across $\beta$-values (trials) to each of the targets. Before examining the correlations of individual fMRI variability magnitudes across targets and arms, we first regressed out the subjects' framewise displacement magnitudes. This ensured that individual fMRI variability measures were not generated by potential differences in head motion (Power et al., 2012).

Correlations. We used Pearson correlation coefficients to assess whether individual kinematic variability magnitudes were correlated across targets, arms, and different kinematic components. Equivalent analyses were performed to determine whether individual fMRI variability magnitudes (in each of the examined ROIs) were correlated across targets and arms, as well as between the variability of each kinematic component and fMRI variability in each ROI. We assessed the statistical significance using permutation tests. We randomly shuffled the variability values of the different subjects in each correlation analysis and computed the correlation. This process was repeated 5000 times to generate 5000 correlation values that represented a distribution of correlations expected by chance (null distribution). For the true (unshuffled) value to be considered significant, it had to surpass the 97.5th percentile of the null distribution (i.e., the equivalent of a $p<0.05$ in a two-tailed $t$ test). We used the false discovery rate (FDR) correction (Benjamini and Hochberg, 1995; Yekutieli and Benjamini, 1999) to correct for the multiple comparisons across target pairs and across ROIs.

Searchlight analysis. In addition to the ROI analysis, we used a searchlight analysis (Kriegeskorte et al., 2006) to map the correlations between fMRI variability and kinematic variability (i.e., movement extent, peak velocity, or direction) throughout the entire cortex. Clusters of 125 functional voxels were defined using a cube with an edge length of five voxels around each gray matter voxel in the native space of each subject. fMRI variability was calculated for each cluster of voxels, as described above in the ROI analysis. After computing the variability map of each subjects, all maps were transformed to a standard cortical surface using Freesurfer and correlation analysis between kinematic and fMRI variabilities were performed for each kinematic measure using movements performed by either the right or left arm. This yielded six correlation maps (three kinematic variables and two arms). A Student's $t$ test was used to determine the significance of the correlation across subjects in each vertex. FDR correction was used to correct for the multiple comparisons performed across vertices (Storey, 2002).

\section{Results}

\section{Intertrial kinematic variability}

Subjects exhibited considerable intertrial kinematic variability in their slice (out-and-back) movements to each of the four targets (Fig. 1B). We focused our analyses on three kinematic components: direction (at endpoint) and extent, which are commonly reported in behavioral studies (Gordon et al., 1994b; Krakauer et al., 2000; van Beers, 2009), and peak velocity, which is commonly reported in electrophysiology studies (Churchland et al., 2006; Cisek, 2006). Note that movement extent and peak velocity are mutually dependent because peak velocity scales with increasing target distance (Gordon et al., 1994a).

Consistent with previous findings, we found that the variance of movement extent and peak velocity grew with the mean (correlation across subjects: $r=0.35$ and $r=0.53$, respectively, averaged across targets and arms). To determine differences in intertrial variability not explained by differences in the mean, we used the CV. In contrast, mean movement direction was not correlated with its SD across trials $(r<0.1)$. There was therefore no reason to normalize this measure, so we used the SD across trials to quantify movement direction variability.

When examining each of the kinematic components separately, individual subjects exhibited consistent magnitudes of intertrial variability across movements to different targets (Fig. $2 A, B)$. Therefore, subjects who were, for example, more variable 
A

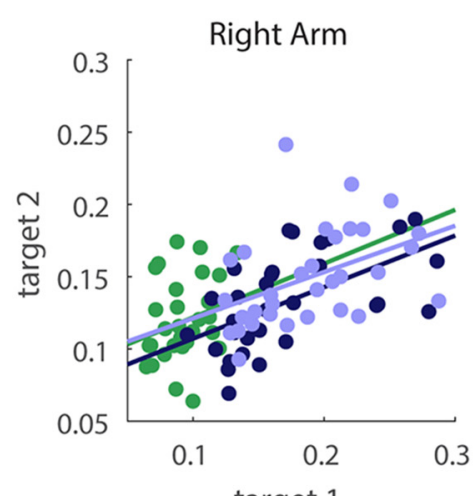

target 1

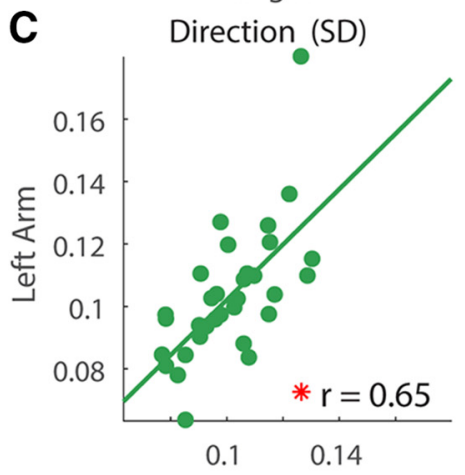

Right Arm

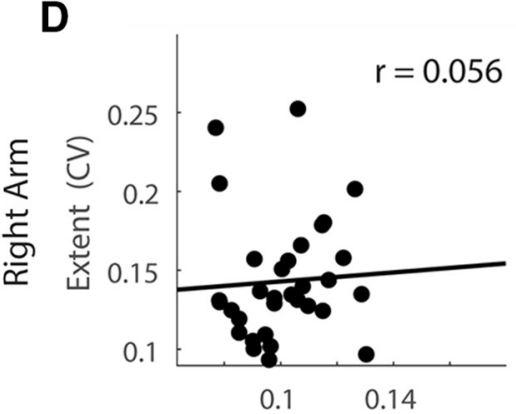

Direction (SD)

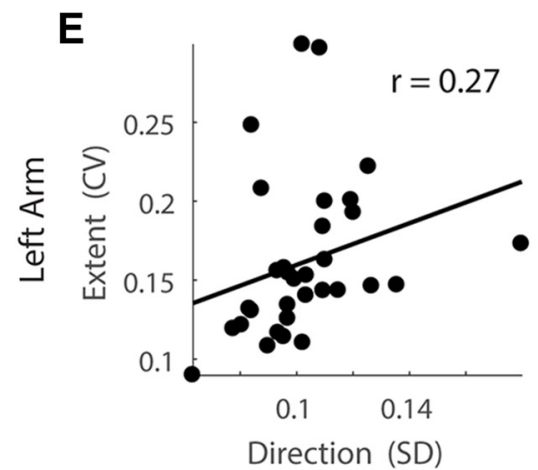

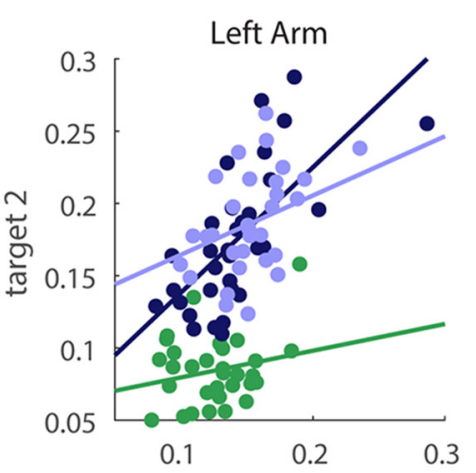

target 1

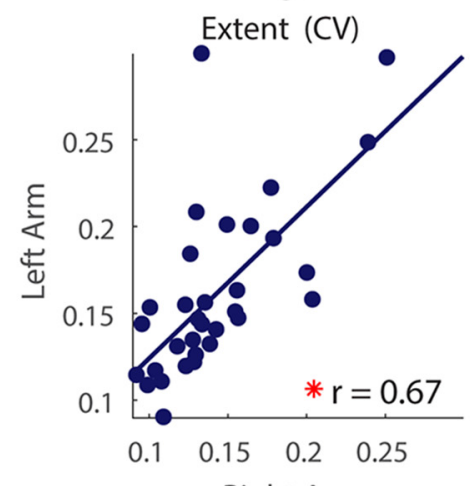

Right Arm

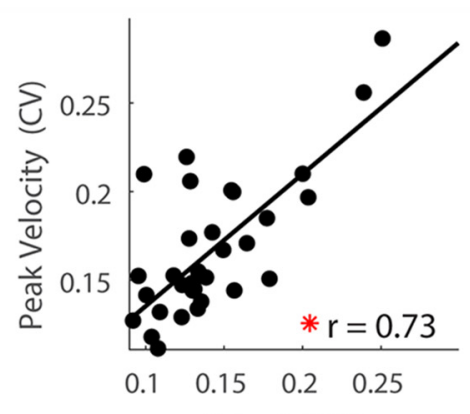

Extent (CV)

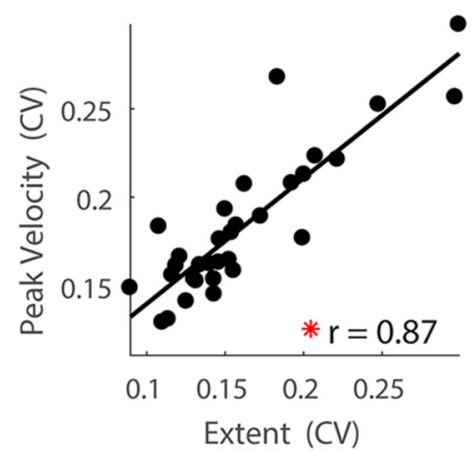

B
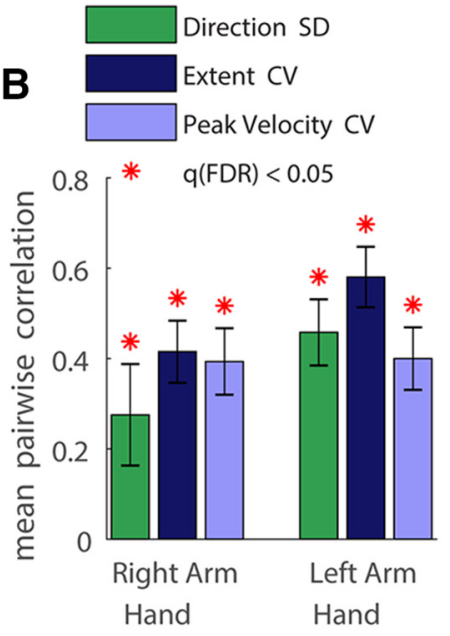

Peak Velocity (CV)
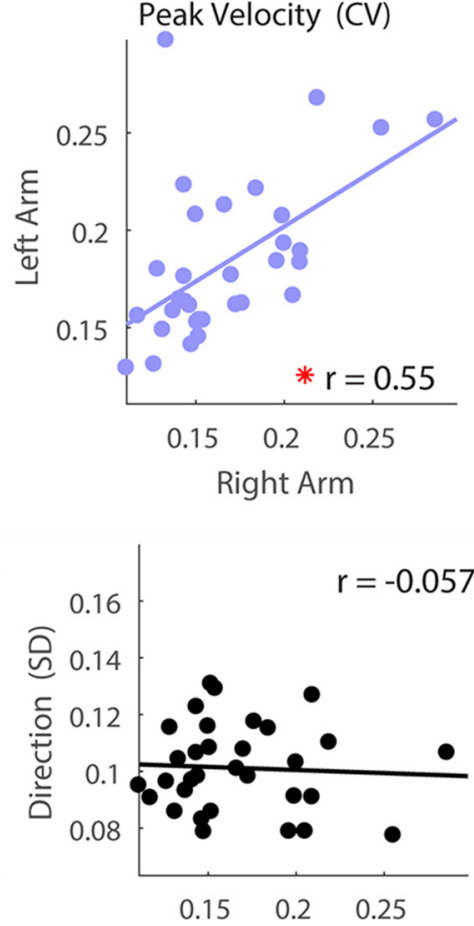

Peak Velocity (CV)

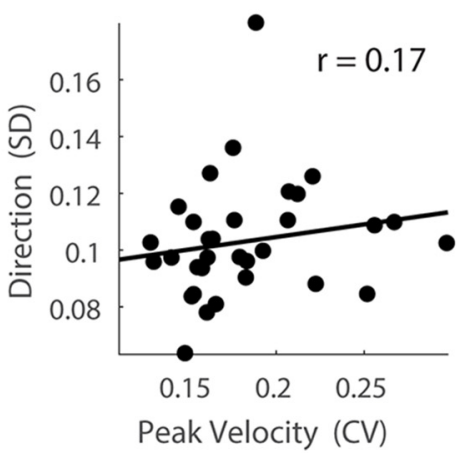

Figure 2. Kinematic variability correlations. We computed the intertrial variability of movement direction (green), extent (dark blue), and peak velocity (light blue) across movements to each target for each of the subjects. $\boldsymbol{A}$, Individual magnitudes of intertrial variability were strongly correlated across the two proximal targets (i.e., regardless of direction). $\boldsymbol{B}$, Means and SEM of the Pearson correlations of the variability across all pairs of targets. Significant correlations are marked with asterisks. C, Scatter plots of the kinematic variability, averaged across targets, of the right and left arms. Each data point represents variability of movements of a single subject. $\boldsymbol{D}, \boldsymbol{E}$, Scatter plots of the kinematic variability, averaged across targets, of the right $(\boldsymbol{D})$ and the left $(\boldsymbol{E})$ arms. For all scatter plots, data points represent different subjects and lines represent linear fits. Significant correlations are marked with red asterisks. 

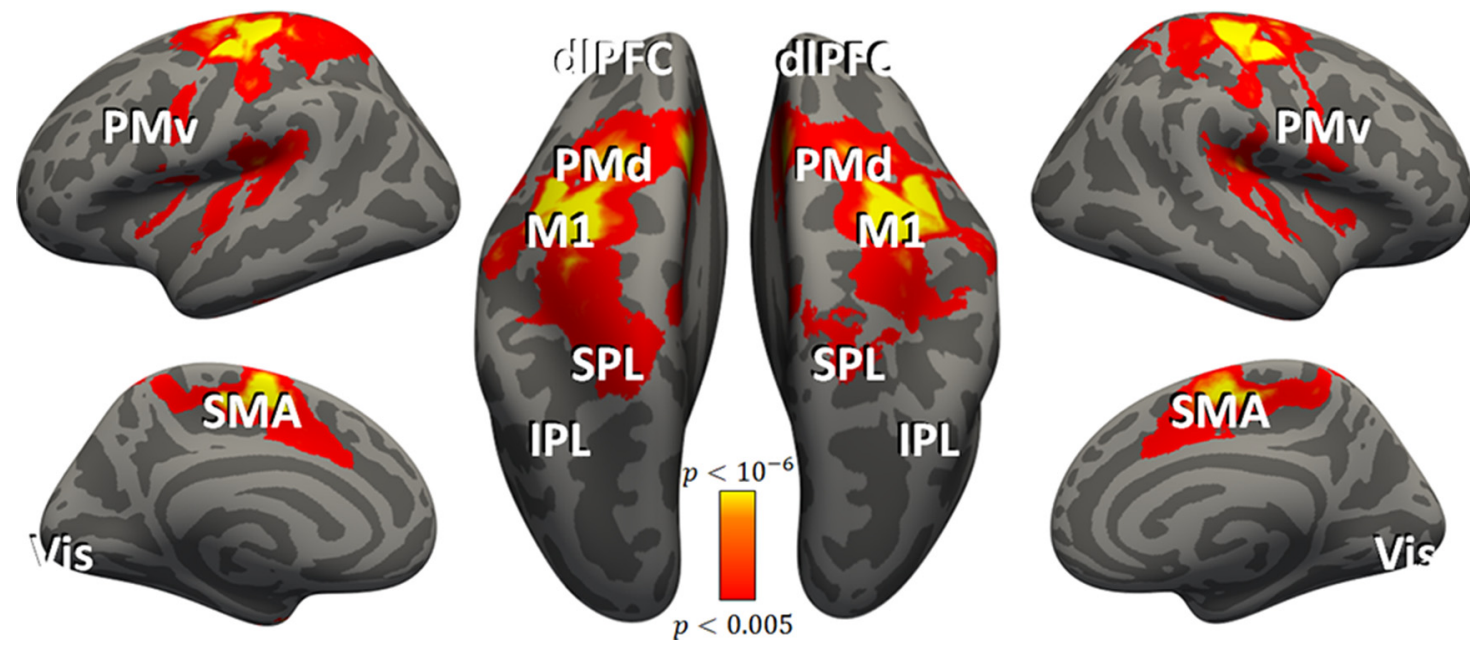

Figure 3. Cortical activation during movement execution. Cortical areas that exhibited larger responses during movement than rest are shown in red/orange. Results were calculated across all subjects (random-effects GLM) and displayed on inflated hemispheres of a template brain. The general locations of the selected ROls are noted (actual ROls were anatomically and functionally defined in each subject; see Materials and Methods): M1, PMd, PMv, SMA, IPL, SPL, dIPFC, and Vis.

in their movement extents to one target tended to be more variable in their movement extents to all other targets. We quantified this by computing the mean Pearson correlation coefficients across all target pairs for movements performed with the right $\operatorname{arm}(r=0.29,0.41$, and 0.39 for movement direction, extent, and peak velocity, respectively, $\left.q_{\text {(FDR })}<0.001\right)$ and left $\operatorname{arm}(r=0.46$, 0.58 , and 0.40 for movement direction, extent, and peak velocity respectively, $\left.q_{\text {(FDR) }}<0.001\right)$. Significant correlations were also evident when comparing the variability magnitudes of each kinematic component across arms (Fig. 2C). For example, subjects with more variable movement extents in right arm movements exhibited more variable movement extents in left arm movements as well ( $r=0.65,0.67$, and 0.55 for movement direction, extent, and peak velocity, respectively, $p<0.001$ ).

Consistent with previous reports (Gordon et al., 1994a), intertrial variability of movement extent and peak velocity were strongly correlated in movements of the right arm $(r=0.73, p<$ 0.001; Fig. $2 D)$ and left $\operatorname{arm}(r=0.87, p<0.001$; Fig. $2 E)$, but variability of movement extent and movement direction (right arm: $r=0.06, p=0.37$; left arm: $r=0.27, p=0.07$ ) or peak velocity and movement direction (right arm: $r=-0.06, p=0.62$; left arm: $r=0.17, p=0.17$ ) were not. Therefore, individuals who exhibited large movement extent and peak velocity variabilities did not necessarily exhibit large movement direction variability and vice versa.

\section{Intertrial fMRI variability}

All subjects exhibited robust fMRI responses during the execution of movements, which enabled us to identify six cortical ROIs that are commonly examined in motor system studies (Fig. 3): M1, PMd, PMv, SMA, SPL, and IPL. In addition to the motor ROIs, we also identified ROIs in Vis, dlPFC, and outside of the brain (OOB).

We then quantified intertrial fMRI variability in each of the ROIs separately for each subject in the following manner: First, we estimated the HRF in each ROI for each target by averaging the fMRI responses across all movements to that target (Fig. 4A). We then used the target-specific HRF in a GLM analysis to estimate a response amplitude/ $\beta$-value for each trial/movement in the experiment (Fig. $4 B$ ). Note that using a target-specific HRF enabled us to compute single trial responses/ $\beta$-values relative to the mean HRF of each subject. This approach discounted potential between-subject differences in the mean amplitude and shape of individual HRFs. Finally, we quantified intertrial fMRI variability by computing the SD across $\beta$-values for each of the targets (Fig. $4 B, C$ ).

Intertrial fMRI variability was correlated across all pairs of targets in most of the ROIs examined (Fig. 5A). Therefore, subjects who exhibited more variable brain responses when moving to one target also exhibited more variable brain responses when moving to other targets. During right arm movements, all ROIs in the left hemisphere except dIPFC and all ROIs in the right hemisphere except PMd and dlPFC exhibited significant pairwise correlations across targets $\left(r>0.32, q_{(\mathrm{FDR})}<0.05\right)$. Correlations in the dlPFC and OOB ROIs were not significant $(r<0.26$, $q_{\text {(FDR) }}>0.1$ ). Together, these findings demonstrate that correlation in fMRI variability magnitudes across targets was specific to cortical ROIs that were activated by the task. Note that early visual cortex was weakly activated in this task by the presentation of the target location at the beginning of each trial and the presentation of the go cue $5 \mathrm{~s}$ later. The significant correlations across targets in early visual cortex demonstrate that some subjects exhibited larger intertrial fMRI variability in visual cortex than others regardless of the movement's target. We demonstrated this phenomena recently (Arazi et al., 2017a, 2017b). Similar results were also apparent for left arm movements (data not shown).

Individual magnitudes of fMRI variability were also significantly correlated across right and left arm movements in many of the examined motor ROIs (Fig. $5 B$ ). This was evident in all ROIs in the left hemisphere $\left(r>0.43, q_{\text {(FDR }}<0.05\right.$; Fig. $5 B$, red bars $)$ except for M1 and dIPFC and in the SPL, PMd, and SMA in the right hemisphere $\left(r>0.47, q_{(\mathrm{FDR})}<0.05\right.$; Fig. $5 B$, yellow bars $)$. In addition, fMRI variability magnitudes were significantly correlated across left and right arm movements in contralateral SPL, PMd, and SMA ROIs $\left(r>0.48, q_{\text {(FDR })}<0.05\right.$; Fig. $5 B$, purple bars). This means that, for example, variability in left PMd during right arm movements was significantly correlated with variability in right PMd during left arm movements. Note that consistent fMRI variability across targets and hands was mostly apparent in parietal and prefrontal motor areas, yet was entirely absent in M1. Correlations in the dlPFC and OOB ROIs were not significant $\left(r<0.33, q_{\text {(FDR) }}>0.09\right)$. This demonstrates that consistent fMRI 
A

Trial Responses

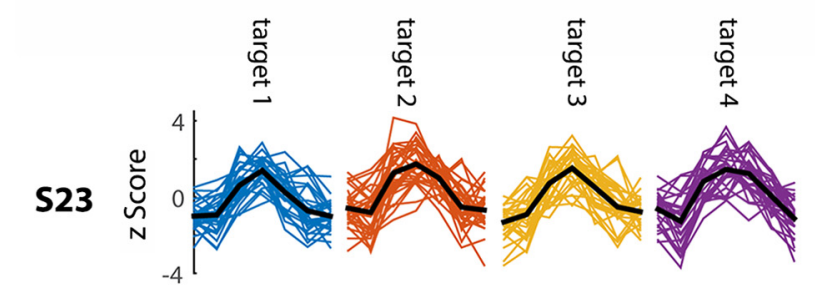

B

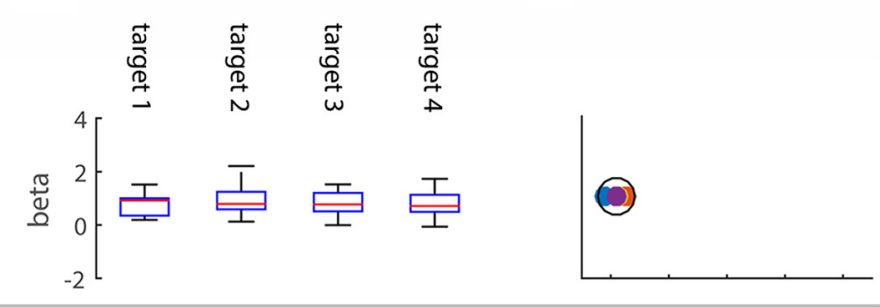

C fMRI Variability
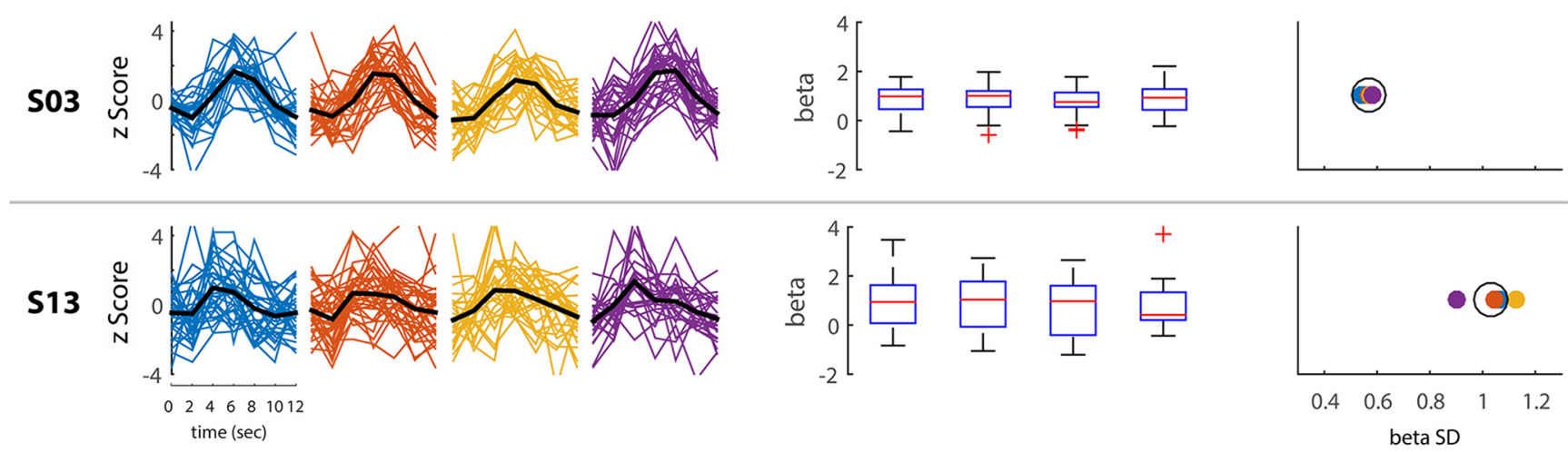

Figure 4. fMRI variability. Examples of intertrial fMRI variability as quantified in left M1 of three subjects during right arm movements are shown. A, Single-trial fMRI responses from left M1 are presented in $z$-scored units color coded according to the different targets; mean HRF across trials (i.e., the HRF used in the GLM analysis) is presented in black. Time point zero corresponds to presentation of the go cue. $\boldsymbol{B}$, Boxplots demonstrating the distributions of $\beta$-values per target. $C, S D$ across $\beta$-values for each target (color code is the same as in $A$ ). The mean SD across targets is represented by the black circle. Each row represents data from a single subject.

variability differences across subjects were not due to differences in scanner measurement noise across subjects. Such scanner noise differences would be apparent in multiple ROIs and even in ROIs located outside the brain.

\section{Relationship between kinematic and fMRI variability}

Subjects with larger intertrial fMRI variability in the IPL exhibited larger intertrial extent variability (Fig. 6). We investigated to what extent between-subject differences in kinematic variability could be explained by fMRI variability measures in right and left ROIs using partial least-squares regression. We performed this analysis separately for right and left hand movements and then averaged across hands. Intertrial fMRI variability in right and left IPL explained $24 \%\left(q_{\text {(FDR) }}=0.004\right)$ of the between-subject differences in extent variability, $15 \%$ of the variability in the peak velocity, and $8 \%$ of the variability in movement direction. The IPL was the only ROI where there was a significant relationship between fMRI variability magnitudes and any of the kinematic variability measures. In contrast, intertrial fMRI variability in M1 explained only $2 \%, 5 \%$, and $4 \%\left(q_{\text {(FDR })}>0.5\right)$ of the betweensubject differences in direction, extent, and peak velocity variability, respectively. Correlations were not significant in any of the control ROIs (dlPFC and OOB, $R^{2}<8 \%, q_{(\mathrm{FDR})}>0.2$ ).

\section{Searchlight analysis}

To examine the spatial selectivity of the cortical-kinematic relationship, we performed an additional analysis using a wholebrain searchlight approach (Kriegeskorte et al., 2006). We mapped the correlations between kinematic variability magnitudes and fMRI variability magnitudes across the entire cortical surface so as not to restrict the analysis to a priori ROIs. We used a volumetric searchlight cube of 125 functional voxels in the cortical gray matter segmented within the native space of each subject. For each searchlight cube, we calculated the intertrial fMRI variability (as described above for the ROIs) and then registered the resulting variability maps of all subjects to a common inflated brain. We calculated Pearson correlation coefficients to estimate the relationship between intertrial fMRI variability magnitudes and variability magnitudes of each kinematic variable: movement extent, peak velocity, and direction.

This analysis yielded three searchlight maps that revealed results complementary to those described above. We did not find any cortical areas where fMRI variability magnitudes were significantly correlated with variability magnitudes in movement direction or peak velocity. Significant positive correlations, however, were found in bilateral inferior parietal cortex when examining movement extent (Fig. 7). Note that the searchlight map is highly symmetric across hemispheres and is relatively similar across movements of the right (Fig. 7, red) and left (Fig. 7, blue) arms.

\section{Alternative sources of fMRI variability}

Between-subject differences in fMRI variability can be generated by several non-neural sources that need to be considered. First, previous studies of fMRI variability have reported that the strength of the mean fMRI response was correlated with the magnitude of intertrial variability across subjects (He, 2013; Ferri et al., 2015). To measure intertrial fMRI variability in individual subjects independently of their mean response, we estimated intertrial variability with respect to the mean HRF apparent in each ROI of each subject (see Materials and Methods). This enabled us to compute the relative $\mathrm{fMRI}$ variability with respect to the actual $\mathrm{HRF}$, as opposed to using a canonical HRF that assumes an identical shape and amplitude across subjects. Indeed, when using this method, intertrial fMRI variability was not correlated significantly with mean fMRI response in any of the ROIs $(r<0.15, p>0.1)$.

Second, we regressed out the mean $\mathrm{fMRI}$ time courses of the lateral ventricles and an ROI containing all gray matter voxels (i.e., "global component"). These time courses represent fMRI 


\section{A}
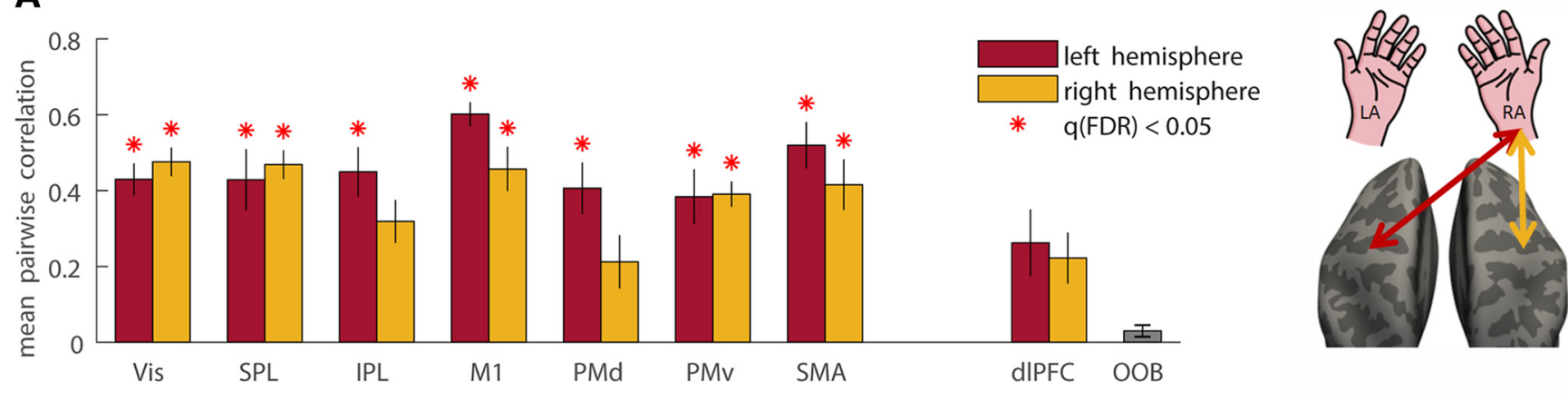

\section{B}
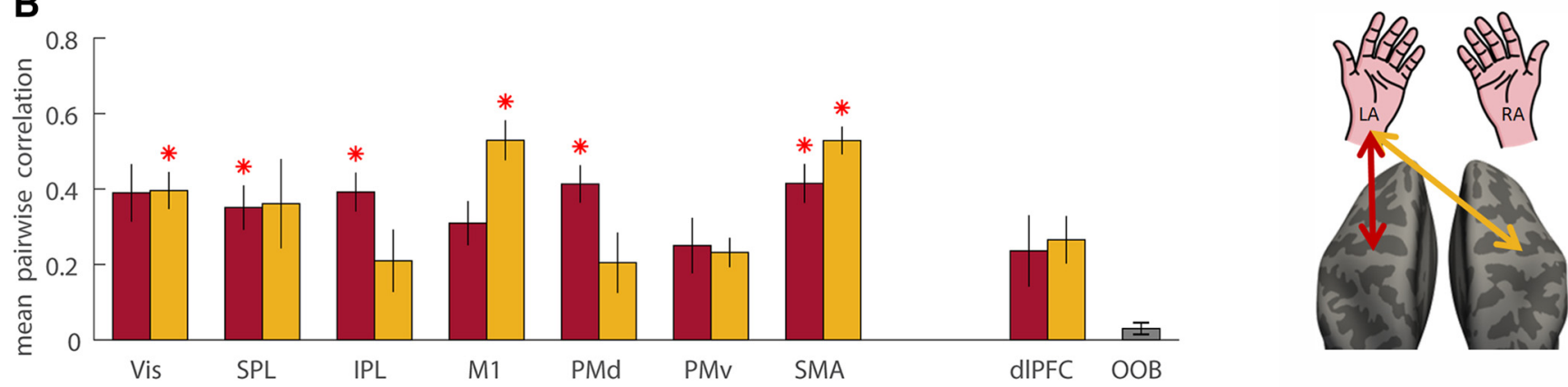

C
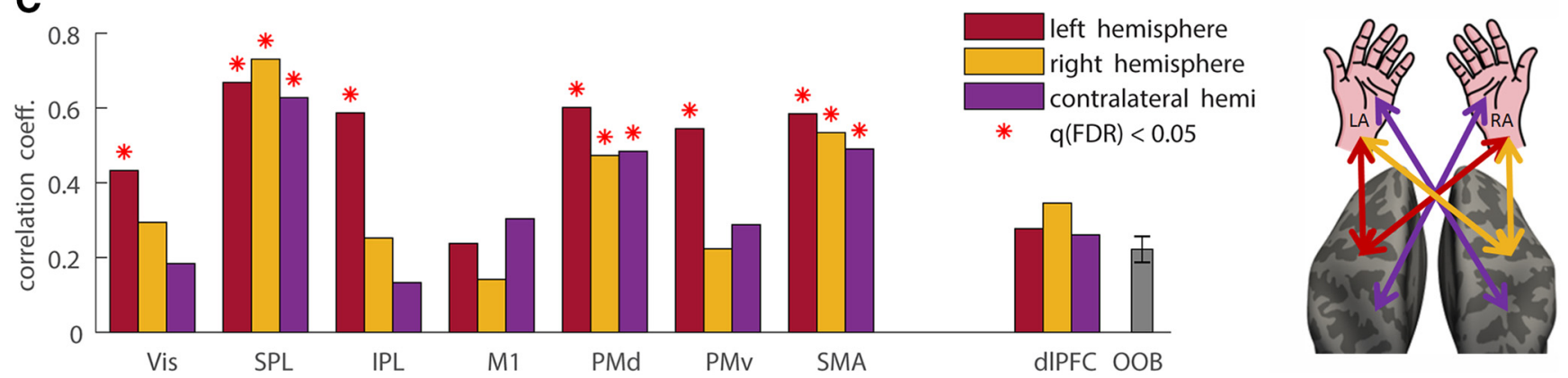

Figure 5. Cortical variability correlations. fMRI variability magnitudes during right $(\boldsymbol{A})$ and left $(\boldsymbol{B})$ arm movements were correlated across all target pairs. Mean pairwise correlation coefficients are presented for each left hemisphere (red) and right hemisphere (yellow) ROI. C, fMRI variability magnitudes were correlated across right and left arm movements in left hemisphere ROIs (red), right hemisphere ROIs (yellow), and contralateral ROls (purple). Significant correlations are marked with red asterisks.

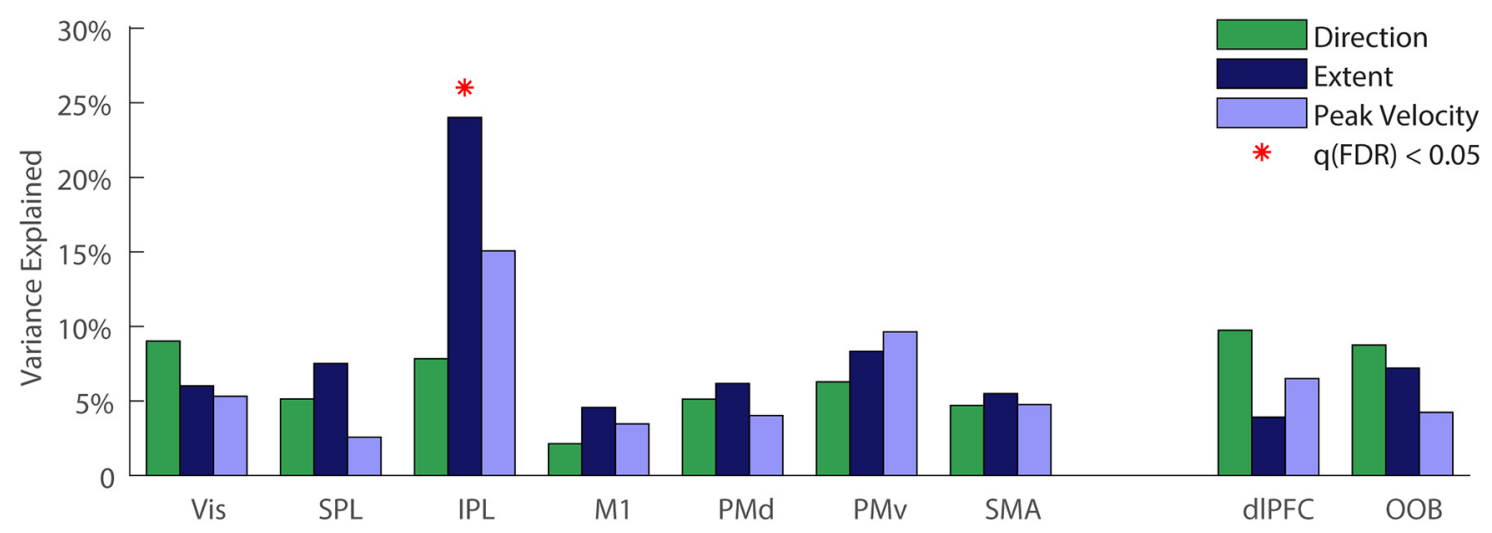

Figure 6. Kinematic variability explained by fMRI variability. Multiple regression was performed between fMRI variability magnitudes in each pair of ROIs (right and left hemispheres) and variability magnitudes of each kinematic variable: direction (green), extent (dark blue), or peak velocity (light blue). This analysis was performed separately for right and left hand movements and the results were averaged. Significant explained variance is marked with red asterisks $\left(q_{(\mathrm{FDR})}<0.05\right)$.

fluctuations that may in part be associated with changes in respiration, blood pressure, and other non-neural origins.

Third, head motion artifacts can generate fMRI variability across trials. To ensure that our results were not generated by head motion artifacts, we regressed out estimated head motion parameters from the fMRI activity of each voxel in the brain before performing the analyses (see Materials and Methods). Furthermore, we also computed the mean framewise displace- 

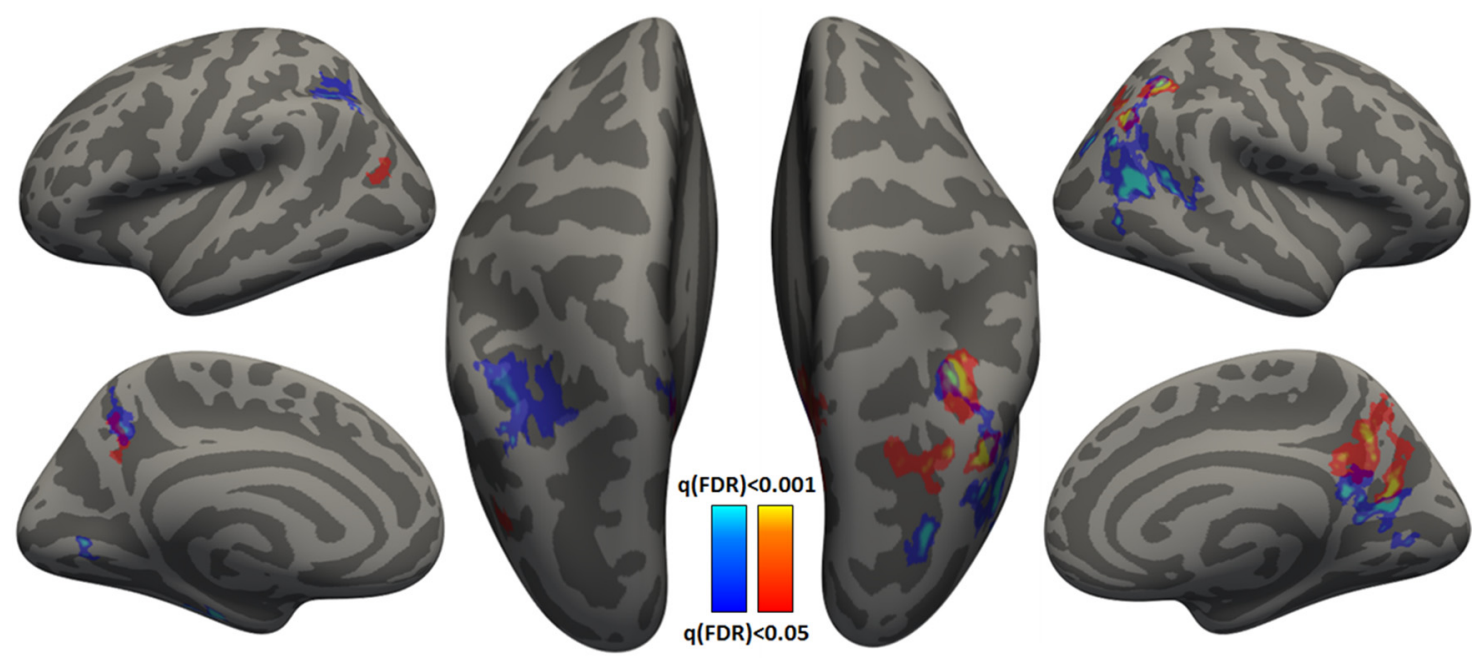

Figure 7. Searchlight analysis displaying cortical areas with significant correlations between movement extent variability and fMRI variability across subjects. Results for right (red) and left (blue) arm movements are presented on the inflated cortical anatomy of a single subject. Correlation significance was determined based on a Student's $t$ test (FDR corrected).

ment across head motion parameters (i.e., the mean amount of head motion across samples/TRs) for each subject. We regressed out individual values of framewise displacement from the fMRI variability magnitudes before examining correlations across targets and/or arms. This ensured that the reported between-subject differences in $\mathrm{fMRI}$ variability magnitudes were not generated by underlying differences in head motion across subjects.

\section{Discussion}

Our results reveal that individual subjects exhibit distinct magnitudes of kinematic variability that are consistent across movements to different locations when performed by either arm. Individual variability magnitudes in movement extent, peak velocity, or direction were strongly correlated across different targets and across arms (Fig. 2). This means that an individual who exhibits large movement extent variability to one target is likely to exhibit large movement extent variability to all other targets regardless of the arm that he/she uses to perform the movements.

Analogous findings were also apparent when examining fMRI variability magnitudes of individual subjects (Fig. 5). Subjects with larger fMRI variability magnitudes in most of the examined motor areas tended to exhibit larger variability regardless of target location or arm used to perform the movements. A surprising exception was M1, where fMRI variability magnitudes were not consistent across arms. This suggests that cortical variability magnitudes in parietal and premotor motor system areas are relatively stable individual characteristics, whereas cortical variability magnitudes in M1 may represent more transient states that change with the choice of effector or task.

The results also revealed a specific relationship between variability magnitudes in one of the kinematic measures, movement extent, and cortical variability magnitudes in one brain area, the IPL. Indeed, fMRI variability magnitudes in the IPL explained $24 \%$ of the differences in movement-extent variability across subjects. In contrast, fMRI variability magnitudes in M1 explained only $5 \%$ of between-subject differences in movementextent variability (Fig. 6). The specificity of these results was further validated by a searchlight analysis that revealed significant correlations between the kinematic and cortical variability magnitudes only with respect to movement extent and only in IPL (Fig. 7). Parietal cortex is thought to play key roles in motor planning, sensory motor mapping, and state estimation (Buneo and Andersen, 2006). Therefore, we suggest that a considerable portion of movement-extent variability is generated by cortical variability associated with movement preparation, rather than cortical variability associated with movement execution.

Note that this is the first study to examine the consistency of kinematic variability across targets/hand and relate it with cortical response variability in humans. Contemporary models of motor control and motor learning (Pekny et al., 2015; Wolpert and Flanagan, 2016) emphasize the importance of intertrial variability for motor system flexibility and accuracy. For example, it has been reported that individuals with larger intertrial behavioral variability learn new motor tasks more quickly (Wu et al., 2014). Note that whereas larger intertrial variability may be useful for flexibility and learning, variability in movement accuracy across trials is often detrimental. Therefore, we speculate that the stable between-subject differences in cortical and kinematic variability magnitudes described here are likely to predispose individual subjects to exhibit different motor capabilities.

\section{Neural sources of kinematic variability}

Previous theories have suggested that intertrial kinematic variability is predominantly generated by the variable activity of sensory neural populations (Osborne et al., 2005; for review, see Lisberger and Medina, 2015), PMd and M1 neural populations involved in motor planning (Churchland et al., 2006), or by neuromuscular variability that characterizes actual movement execution (van Beers et al., 2004; van Beers, 2009). It is entirely possible, however, that different sources of neural variability generate kinematic variability under different experimental conditions such that behavioral motor variability would embody the sum of multiple neural variability sources (for review, see Faisal et al., 2008). With this in mind, neural variability in a particular brain area is likely to explain a certain proportion of kinematic variability. Furthermore, neural variability in different brain areas may generate variability in different kinematic components of movements (e.g., movement extent vs movement direction).

Our results indeed demonstrate that approximately onequarter of the between-subject differences in movement extent variability are explained by individual neural variability differences in parietal cortex, which is thought to play a dominant role in the planning and preparation of reaching movements (Cohen and Andersen, 2002). Although previous electrophysiology stud- 
ies have reported that variability in M1 and PMd neural activity (during preparation for movement) generates variability in peak movement velocity (Churchland et al., 2006; Chaisanguanthum et al., 2014), our results suggest that stronger relationships between neural and kinematic variability will be evident in parietal brain areas and particularly in IPL (Figs. 6, 7).

It may seem surprising that correlations between kinematic variability and fMRI variability were weak in M1 given that it is the lowest area in the cortical motor hierarchy (Shadmehr and Krakauer, 2008). In humans, however, only $30-40 \%$ of the axons in the corticospinal tract originate from neurons in M1; the rest originate from the premotor, supplementary motor, and posterior parietal cortices (Kandel et al., 2013). This means that neural variability in parietal regions may generate kinematic variability downstream of M1 in spinal motor circuits. A potentially interesting analogy can be found in songbirds, in which the lateral magnocellular nucleus of anterior nidopallium has evolved to inject direct neural variability into the motor circuits that control singing, apparently enabling juvenile birds to learn through trial and error (Ölveczky et al., 2011).

Parietal cortex contains neural populations that perform a wide variety of computations that are essential for motor control, including motor planning, sensory-motor mapping, and state estimation (Wolpert and Ghahramani, 2000; Cohen and Andersen, 2002; Buneo and Andersen, 2006; Churchland et al., 2006; Shadmehr and Krakauer, 2008). More specifically, neural populations in the IPL are thought to integrate high-order sensory and motor information in support of high-level motor functions (Fogassi and Luppino, 2005) and represent conscious motor intentions (Desmurget and Sirigu, 2012). Within all of these frameworks, each with its specific mechanistic focus, variability in the activity of parietal neural populations would generate variability in the kinematics of executed movements.

An alternative interpretation of our results might emphasize the sensory roles of parietal cortex. In this case, the causality would be reversed such that the measured fMRI variability would be generated by movement variability (and not the other way around). Although it is difficult to entirely rule this option out, it is important to note that we did not find significant correlations between any of the kinematic measures and fMRI variability magnitudes in somatosensory cortices (Fig. 7). The selectivity of the results to IPL argues against such a sensory-driven explanation of the results.

Finally, it is important to note that we and all previous authors of electrophysiology studies on the topic measured variability only in the kinematics of the movements and not in their dynamics. It is highly possible that intertrial variability in movement dynamics (i.e., muscle activation), which are not necessarily captured in measures of kinematic variability, may be explained by intertrial neural variability in specific brain areas.

\section{Decomposing neural variability}

Neural variability is likely to arise from a wide variety of molecular and cellular mechanisms that govern neural transduction and transmission in addition to mechanisms that govern neural network dynamics. Although it is difficult to disentangle the different sources of neural variability using neuroimaging, it is possible to decompose variability into different spatial and temporal components using measures from different types of neuroimaging and electrophysiological techniques (Dinstein et al., 2015). When studying variability with fMRI, it is possible to quantify intertrial variability simultaneously in multiple different brain areas, but the temporal resolution of this measure is limited by the sluggish nature of the hemodynamic response (Heeger and Ress, 2002). Furthermore, because fMRI is not a direct measure of neural activity, but rather a measure of hemodynamic changes over time, intertrial variability in the function of neurovascular coupling mechanisms will be an inherent part of the fMRI intertrial variability measure. This limits the ability to measure neural variability with fMRI and therefore limits the ability to relate neural variability and behavioral variability measures. With this in mind, it is impressive that we were able to identify a consistent relationship between fMRI variability and movement extent variability that was similarly evident in movements of right and left arm (Figs. 6, 7). We speculate that stronger relationships may be revealed with direct measures of human neural activity such as ECOG recordings.

\section{Hemispheric lateralization}

Although arm movements are clearly generated and controlled by neural activity in the contralateral hemisphere (Penfield and Boldrey, 1937), human fMRI studies show activity and even directional selectivity of arm movement (Fabbri et al., 2010; Haar et al., 2015) across the cortical motor hierarchy in the ipsilateral hemisphere. Here, we found significant correlations between movement extent variability and neural variability in both the contralateral and ipsilateral hemispheres. We speculate that neural variability in both hemispheres may therefore have an impact on the accuracy and reliability of arm movements.

\section{Conclusions}

This study demonstrates that kinematic variability and parietal and prefrontal cortical variability are stable individual traits that appear consistently across movements to different targets when performed by either arm. Furthermore, these variabilities are related such that subjects with larger neural variability in IPL exhibited larger movement-extent variability. We believe that these results represent an important first step for understanding how neural variability may generate movement variability in humans and thereby predispose individuals to exhibit distinct motor capabilities such as motor learning proficiency.

\section{References}

Arazi A, Censor N, Dinstein I (2017a) Neural Variability Quenching Predicts Individual Perceptual Abilities. J Neurosci 37:97-109. CrossRef Medline

Arazi A, Gonen-Yaacovi G, Dinstein I (2017b) Trial-by-trial neural variability is an individual human trait. bioRxiv, 096198. CrossRef

Benjamini Y, Hochberg Y (1995) Controlling the false discovery rate: a practical and powerful approach to multiple testing. J R Stat Soc B 57: 289-300.

Braun DA, Aertsen A, Wolpert DM, Mehring C (2009) Motor Task Variation Induces Structural Learning. Curr Biol 19:352-357. CrossRef Medline

Buneo CA, Andersen RA (2006) The posterior parietal cortex: Sensorimotor interface for the planning and online control of visually guided movements. Neuropsychologia 44:2594-2606. CrossRef Medline

Chaisanguanthum KS, Shen HH, Sabes PN (2014) Motor variability arises from a slow random walk in neural state. J Neurosci 34:12071-12080. CrossRef Medline

Churchland MM, Abbott LF (2012) Two layers of neural variability. Nat Neurosci 15:1472-1474. CrossRef Medline

Churchland MM, Afshar A, Shenoy KV (2006) A Central Source of Movement Variability. Neuron 52:1085-1096. CrossRef Medline

Cisek P (2006) Preparing for speed. Focus on "Preparatory activity in premotor and motor cortex reflects the speed of the upcoming reach." J Neurophysiol 96:2842-2843. CrossRef Medline

Cohen YE, Andersen RA (2002) A common reference frame for movement 
plans in the posterior parietal cortex. Nat Rev Neurosci 3:553-562. CrossRef Medline

Desmurget M, Sirigu A (2012) Conscious motor intention emerges in the inferior parietal lobule. Curr Opin Neurobiol 22:1004-1011. CrossRef Medline

Destrieux C, Fischl B, Dale A, Halgren E (2010) Automatic parcellation of human cortical gyri and sulci using standard anatomical nomenclature. Neuroimage 53:1-15. CrossRef Medline

Dinstein I, Heeger DJ, Behrmann M (2015) Neural variability: friend or foe? Trends Cogn Sci 19:322-328. CrossRef Medline

Fabbri S, Caramazza A, Lingnau A (2010) Tuning curves for movement direction in the human visuomotor system. J Neurosci 30:13488-13498. CrossRef Medline

Faisal AA, Selen LP, Wolpert DM (2008) Noise in the nervous system. Nat Rev Neurosci 9:292-303. CrossRef Medline

Faul F, Erdfelder E, Buchner A, Lang AG (2009) Statistical power analyses using $G^{\star}$ Power 3.1: tests for correlation and regression analyses. Behav Res Methods 41:1149-1160. CrossRef Medline

Ferri F, Costantini M, Huang Z, Perrucci MG, Ferretti A, Romani GL, Northoff G (2015) Intertrial variability in the premotor cortex accounts for individual differences in peripersonal space. J Neurosci 35:1632816339. CrossRef Medline

Fischl B (2012) FreeSurfer. Neuroimage 62:774-781. CrossRef Medline

Fogassi L, Luppino G (2005) Motor functions of the parietal lobe. Curr Opin Neurobiol 15:626-631. CrossRef Medline

Gordon J, Ghilardi MF, Cooper SE, Ghez C (1994a) Accuracy of planar reaching movements. II. Systematic extent errors resulting from inertial anisotropy. Exp Brain Res 99:112-130. CrossRef Medline

Gordon J, Ghilardi MF, Ghez C (1994b) Accuracy of planar reaching movements. I. Independence of direction and extent variability. Exp Brain Res 99:97-111. CrossRef Medline

Haar S, Donchin O, Dinstein I (2015) Dissociating visual and motor directional selectivity using visuomotor adaptation. J Neurosci 35:6813-6821. CrossRef Medline

Harbourne RT, Stergiou N (2009) Movement variability and the use of nonlinear tools: principles to guide physical therapist practice. Phys Ther 89:267-282. CrossRef Medline

He BJ (2013) Spontaneous and task-evoked brain activity negatively interact. J Neurosci 33:4672-4682. CrossRef Medline

Heeger DJ, Ress D (2002) What Does fMRI Tell Us About Neuronal Activity? Nat Rev Neurosci 3:142-151. CrossRef Medline

Herzfeld DJ, Shadmehr R (2014) Motor variability is not noise, but grist for the learning mill. Nat Neurosci 17:149-150. CrossRef Medline

Kandel E, Schwartz J, Jessell T, Siegelbaum SA, Hudspeth AJ (2013) Principles of neural science. Fifth Edition. New York: McGraw Hill Professional.

Kao MH, Doupe AJ, Brainard MS (2005) Contributions of an avian basal ganglia-forebrain circuit to real-time modulation of song. Nature 433: 638-643. CrossRef Medline

Krakauer JW, Pine ZM, Ghilardi MF, Ghez C (2000) Learning of visuomotor transformations for vectorial planning of reaching trajectories. J Neurosci 20:8916-8924. Medline

Kriegeskorte N, Goebel R, Bandettini P (2006) Information-based functional brain mapping. Proc Natl Acad Sci U S A 103:3863-3868. CrossRef Medline

Latash ML, Scholz JP, Schöner G (2007) Toward a new theory of motor synergies. Motor Control 11:276-308. CrossRef Medline
Lisberger SG, Medina JF (2015) How and why neural and motor variation are related. Curr Opin Neurobiol 33:110-116. CrossRef Medline

Mandelblat-CerfY, Paz R, Vaadia E (2009) Trial-to-trial variability of single cells in motor cortices is dynamically modified during visuomotor adaptation. J Neurosci 29:15053-15062. CrossRef Medline

Ölveczky BP, Otchy TM, Goldberg JH, Aronov D, Fee MS (2011) Changes in the neural control of a complex motor sequence during learning. J Neurophysiol 106:386-397. CrossRef Medline

Osborne LC, Lisberger SG, Bialek W (2005) A sensory source for motor variation. Nature 437:412-416. CrossRef Medline

Pekny SE, Izawa J, Shadmehr R (2015) Reward-dependent modulation of movement variability. J Neurosci 35:4015-4024. CrossRef Medline

Penfield W, Boldrey E (1937) Somatic motor and sensory representation in the cerebral cortex of man as studies by electrical stimulation. Brain 60: 389-443. CrossRef

Power JD, Barnes KA, Snyder AZ, Schlaggar BL, Petersen SE (2012) Spurious but systematic correlations in functional connectivity MRI networks arise from subject motion. Neuroimage 59:2142-2154. CrossRef Medline

Sauerbrei BA, Lubenov EV, Siapas AG (2015) Structured variability in Purkinje cell activity during locomotion. Neuron 87:840-852. CrossRef Medline

Schmidt RA, Zelaznik H, Hawkins B, Frank JS, Quinn JT Jr (1979) Motoroutput variability: a theory for the accuracy of rapid motor acts. Psychol Rev 47:415-451. Medline

Shadmehr R, Krakauer JW (2008) A computational neuroanatomy for motor control. Exp Brain Res 185:359-381. CrossRef Medline

Stein RB, Gossen ER, Jones KE (2005) Neuronal variability: noise or part of the signal? Nat Rev Neurosci 6:389-397. Medline

Storey JD (2002) A direct approach to false discovery rates. J R Stat Soc Ser B Stat Methodol 64:479-498. CrossRef

Teo JT, Swayne OB, Cheeran B, Greenwood RJ, Rothwell JC (2011) Human theta burst stimulation enhances subsequent motor learning and increases performance variability. Cereb Cortex 21:1627-1638. CrossRef Medline

Todorov E (2004) Optimality principles in sensorimotor control. Nat Neurosci 7:907-915. CrossRef Medline

van Beers RJ (2009) Motor learning is optimally tuned to the properties of motor noise. Neuron 63:406-417. CrossRef Medline

van Beers RJ, Haggard P, Wolpert DM (2004) The role of execution noise in movement variability. J Neurophysiol 91:1050-1063. Medline

Wilson C, Simpson SE, van Emmerik RE, Hamill J (2008) Coordination variability and skill development in expert triple jumpers. Sports Biomech 7:2-9. CrossRef Medline

Wolpert DM, Flanagan JR (2016) Computations underlying sensorimotor learning. Curr Opin Neurobiol 37:7-11. CrossRef Medline

Wolpert DM, Ghahramani Z (2000) Computational principles of movement neuroscience. Nat Neurosci 3:1212-1217. CrossRef Medline

Woolley SC, Kao MH (2015) Variability in action: Contributions of a songbird cortical-basal ganglia circuit to vocal motor learning and control. Neuroscience 296:39-47. CrossRef Medline

Wu HG, Miyamoto YR, Gonzales Castro LN, Ölveczky BP, Smith MA (2014) Temporal structure of motor vriability is dynamically regulated and predicts motor learning ability. Nat Neurosci 17:312-321. CrossRef Medline

Yekutieli D, Benjamini Y (1999) Resampling-based false discovery rate controlling multiple test procedures for correlated test statistics. J Stat Plan Inference 82:171-196. CrossRef 